\title{
Pulmonary Manifestation of Novel Swine-Origin Influenza A (H1N1) Virus (S-OIV) Infection in Immunocompromised Patients: Initial Findings with Multidetector Computed Tomography
}

\author{
A. Laqmani G. Adam M. Regier
}

Center for Radiology and Endoscopy, Department of Diagnostic and Interventional Radiology, University Medical

Center Hamburg-Eppendorf, Hamburg, Germany

\section{Key Words}

H1N1 - Swine-origin influenza A - Immunocompression •

Multidetector computed tomography

\begin{abstract}
Objective: To describe initial multidetector computed tomographic (MDCT) findings of novel swine-origin influenza A (H1N1) virus (S-OIV) infection in immunocompromised patients and to evaluate whether or not identification of certain abnormalities can help predict patients who are at risk for a severe clinical course. Subjects and Methods: This retrospective study included 13 patients with confirmed S-OIV infection suffering from an underlying immunodeficiency or who were receiving immunosuppressive therapy. All patients underwent MDCT of the thorax due to respiratory distress. All data were read by two independent radiologists who described the type and pattern of opacities, distribution and extent of the abnormalities observed. Adverse outcome measures were defined as acute respiratory distress syndrome with the need for mechanical ventilation, extracorporeal membrane oxygenation or death. Results: MDCT revealed pulmonary manifestations in 12 (92\%) of 13 individuals. Six (50\%) patients showed an adverse outcome with development of acute respiratory distress syndrome, 4 of
\end{abstract}

these died. The most common findings were ground-glass opacities $(10 / 12 ; 83 \%)$ and pulmonary consolidation (7/12; $58 \%)$ predominantly with a bilateral distribution. Reticular pattern and a tree-in-bud appearance were found in 3/12 $(25 \%)$, respectively. Bilateral opacities with extensive involvement of the lung parenchyma were most predictive of a severe clinical course. Conclusion: The MDCT scan in immunocompromised patients with confirmed S-OIV infection frequently revealed pulmonary abnormalities, which included ground-glass opacities and consolidations. Therefore, prediction of an adverse clinical outcome could be made in patients with MDCT findings demonstrating bilateral extensive consolidations, often combined with ground-glass opacities.

Copyright $\odot 2012$ S. Karger AG, Basel

\section{Introduction}

Over the past 2 years the highly contagious novel swine-origin influenza A (H1N1) virus (S-OIV) has rapidly spread worldwide, causing a new global pandemic. In June 2009, the World Health Organization raised the pandemic alert to phase 6 , indicating a global pandemic with widespread community transmission on at least two

\section{KARGER \\ Fax +4161306 1234 \\ E-Mail karger@karger.ch}

www.karger.com (c) 2012 S. Karger AG, Basel

$1011-7571 / 12 / 0216-0548 \$ 38.00 / 0$

Accessible online at:

www.karger.com/mpp
Azien Laqmani, MD

Center for Radiology and Endoscopy, Department of Diagnostic and Interventional Radiology, University Medical Center Hamburg-Eppendorf

Martinistrasse 52, DE-20246 Hamburg (Germany)

Tel. +49 40741054 029, E-Mail a.laqmani@uke.de 
continents [1]. Although the majority of cases revealed a mild, self-limited course, severe illness with an adverse outcome including progressive respiratory failure, acute respiratory distress syndrome and death was described in younger individuals, pregnant women, patients with chronic lung disease, and immunocompromised patients [2-5]. As August 1, 2010 over 18,449 deaths among laboratory-confirmed cases of S-OIV had been reported to the World Health Organization [6]. In 2009, a large study of patients with S-OIV infection showed that $18 \%$ of adult hospitalized patients were immunocompromised, either due to immunodeficiency or immunosuppressive medication [4].

Recent reports demonstrated that immunocompromised individuals such as patients following hematopoietic stem cell transplantation and those receiving chemotherapy due to leukemia are at an increased risk for influenza and a more severe and prolonged course $[7,8]$.

So far, only little is known about the imaging findings in immunocompromised patients suffering from respiratory affection of S-OIV $[8,9]$. Therefore, the purpose of this study was to describe the initial pulmonary multidetector computed tomographic (MDCT) findings of SOIV infection in immunocompromised patients and to determine whether or not identification of certain abnormalities can help predict patients who are at risk for a severe clinical course.

\section{Subjects and Methods}

\section{Study Population}

As this was a retrospective study written informed consent was waived. First, between June 2009 and February 2010 the archive of the Department of Medical Microbiology, Virology and Hygiene was screened for patients with positive results by realtime reverse transcriptase polymerase chain reaction tests of respiratory swabs for S-OIV. This resulted in 231 individuals. Further, this subset was reduced to patients with underlying immunocompression. Patients were considered immunocompromised if they suffered from hematological malignancies or human immunodeficiency virus (HIV) infection, had received hematopoietic stem cell or solid organ transplantation, or if they were receiving immunosuppressive therapy including corticosteroids. Therefore, 14 of the 231 patients were identified as being immunocompromised and having undergone thoracic MDCT. One of these 14 patients was excluded because of concomitant pulmonary invasive aspergillosis, thus the patient sample for the present study was reduced to 13 immunocompromised patients.

The underlying immunocompromising medical conditions of the patient sample were as follows: hematological malignancies $(n=9)$ including 4 patients who had already received hematopoietic stem cell transplantation. All were diagnosed with S-OIV infection before day 100 after transplantation. Four patients with hematological malignancies were receiving chemotherapy. One patient suffered from an acquired immunodeficiency syndrome. Three patients were immunocompromised due to immunosuppressive therapy with cyclosporine, everolimus, methotrexate, azathioprine or long-term oral corticosteroid therapy. The administered immunosuppressive therapy was given for solid organ transplantation $(\mathrm{n}=2)$ and chronic obstructive pulmonary disease $(n=1)$. The underlying disease of 1 of the patients with the organ transplant was Wegener's granulomatosis. Six patients were lymphopenic, 4 neutropenic and another 4 leukopenic.

The study population consisted of 9 men and 4 women ranging in age from 43 to 68 (mean age 59 years).

\section{MDCT Image Acquisition}

MDCT scans were performed on average 7 days after the onset of symptoms (range, 4-11 days). Thoracic MDCT studies were performed using a 256-slice MDCT scanner (Brilliance iCT, Philips, Best, The Netherlands) in standard resolution mode. Highresolution mode was not available for the studies. The image acquisition parameters were chosen as follows: tube voltage $120 \mathrm{kV}$; tube current time product 150-200 mAs; rotation time $500 \mathrm{~ms}$; pitch 0.993 and slice thickness $5 \mathrm{~mm}$. Nine MDCT studies were performed without contrast and $0.6-\mathrm{mm}$ reformats were obtained. In 4 patients $70 \mathrm{ml}$ of an iodine-based contrast agent (Imeron 300, Bracco Imaging, Germany) was administered at an injection rate of $4 \mathrm{ml} / \mathrm{s}$.

\section{Image Analysis}

Using a standard PACS viewer (Centricity PACS Viewer, GE Healthcare, Munich, Germany) two radiologists (A.L. and M.R.) consensually reviewed all MDCTs. They were blinded to the clinical symptoms and outcomes of the patients. All images were assessed for the presence of several abnormal patterns, which were further subclassified according to the definitions of the Fleischner Society glossary as ground-glass opacities (hazy increased opacity with preservation of bronchial and vascular margins), consolidations (increased opacity obscuring bronchial and vascular margins), nodules (rounded focal opacities $<3 \mathrm{~cm}$ ), reticular pattern (linear opacities forming a meshlike pattern) and tree-inbud pattern (centrilobular branching structures) [10]. The presence of an air bronchogram was also noted. The distribution was divided into unilateral, bilateral, central, peribronchovascular or subpleural. The extent of the findings was graded into 0-25, 26$50,51-75,76-100 \%$ of lung parenchyma. Enlargement of mediastinal or hilar lymph nodes and pleural effusion was also noted.

The pattern and distribution of the findings were further analyzed with respect to the clinical course and outcome. Adverse outcome measures were defined as the need for mechanical ventilation, extracorporeal membrane oxygenation therapy or the occurrence of death.

\section{Statistical Analysis}

Quantitative parameters were reported as the mean \pm standard deviation. For categorical variables, the percentages of patients in each category were calculated. Differences in the two groups were tested by using Fisher's exact test. A two-tailed p value of less than 0.05 indicated a significant difference. All of the data were analyzed with statistical software (SPSS, version 20.0; SPSS, Chicago, Ill., USA). 


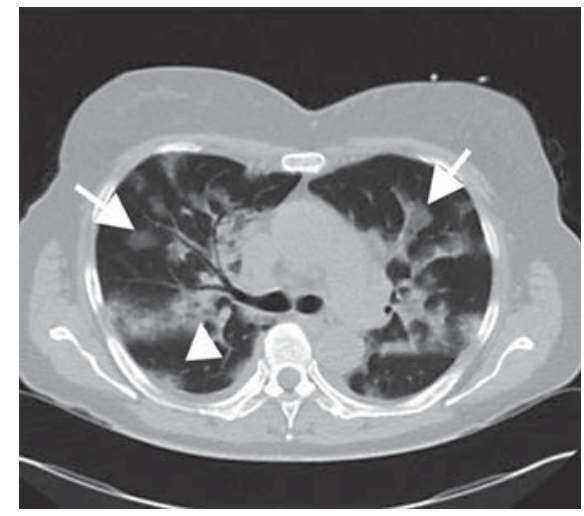

Fig. 1. A 64-year-old female patient with multiple myeloma s/p stem cell transplantation. Axial MDCT scan substantiates the bilateral presence of consolidations (arrowhead) with positive air bronchogram as well as ground-glass opacities (arrows).

\section{Results}

Of the 13 immunocompromised patients, 12 (92\%) revealed abnormalities on the initial MDCT. Of 12 patients with an abnormal MDCT, 6 (50\%) showed an adverse outcome with respiratory failure and acute respiratory distress syndrome, 4 of these died. The remaining 6 of the 12 patients with initial abnormal imaging findings had a mild clinical course.

\section{MDCT Findings}

Of the 12 immunocompromised patients with abnormal MDCT findings, 10 (83\%) were dominated by ground-glass opacities. Consolidation was revealed in 7 (58\%) patients. A combination of both patterns was seen in $6(50 \%)$ patients (fig. 1). MDCT findings are summarized in table 1.

An air bronchogram is included in the majority of the MDCT scans (10/12, 83\%). Three (25\%) patients with abnormal findings revealed centrilobular nodules in a treein-bud pattern. Nodular opacities were seen in only 2 (17\%) patients. All 12 patients demonstrated subpleural predominance of abnormalities; of the 12 patients 11 (92\%) showed a peribronchovascular distribution. The detected abnormalities were bilateral in $10(83 \%)$, predominantly affecting the lower lung in 9 (75\%), and the middle lung zone in 7 (58\%). Five (42\%) patients showed an involvement of more than $50 \%$ of the lung parenchyma, including $33 \%(4 / 12)$ of patients, in whom $>75 \%$ of the lung parenchyma was affected. Nodal enlargement was depicted in 5 (42\%) patients. Pleural effusion was seen in $4(33 \%)$ patients.
Table 1. Initial abnormal MDCT findings of S-OIV infection in immunocompromised patients

\begin{tabular}{lcll}
\hline Characteristic & $\begin{array}{l}\text { Initial } \\
\text { abnormal } \\
\text { MDCT }(\mathrm{n}=12)\end{array}$ & $\begin{array}{l}\text { Favorable } \\
\text { outcome } \\
(\mathrm{n}=6)\end{array}$ & $\begin{array}{l}\text { Adverse } \\
\text { outcome } \\
(\mathrm{n}=6)\end{array}$ \\
\hline $\begin{array}{lll}\text { Opacity } \\
\text { Consolidation }\end{array}$ & $7(58)$ & $2(33)$ & $5(83)$ \\
Ground-glass & $10(83)$ & $5(83)$ & $5(83)$ \\
Consolidation + & & & \\
$\quad$ ground glass & $6(50)$ & $2(33)$ & $4(67)$ \\
Nodules & $2(17)$ & $1(17)$ & $1(17)$ \\
Reticular pattern & $3(25)$ & $2(33)$ & $1(17)$ \\
Tree-in-bud & $3(25)$ & $3(50)$ & $0(0)$ \\
Positive air bronchogram & $10(83)$ & $4(67)$ & $6(100)$ \\
Extent & & & \\
1-25\% & $3(25)$ & $2(33)$ & $1(17)$ \\
26-50\% & $4(33)$ & $3(50)$ & $1(17)$ \\
51-75\% & $1(8)$ & $1(17)$ & $0(0)$ \\
$76-100 \%$ & $4(33)$ & $0(0)$ & $4(67)$ \\
Upper lobe & $4(33)$ & $1(17)$ & $3(50)$ \\
Middle lobe & $7(58)$ & $2(33)$ & $5(83)$ \\
Lower lobe & $9(75)$ & $3(50)$ & $6(100)$ \\
Distribution & & & \\
Unilateral & $2(17)$ & $2(33)$ & $0(0)$ \\
Bilateral & $6(83)$ & $4(67)$ & $6(100)$ \\
Central & $11(92)$ & $1(17)$ & $5(83)$ \\
Peribronchovascular & $5(83)$ & $6(100)$ \\
Subpleural & $12(100)$ & $6(100)$ & $6(100)$ \\
Lymph node & $2(42)$ & $2(33)$ & $3(50)$ \\
Pleural effusion & $2(33)$ & $2(33)$ \\
\hline
\end{tabular}

Figures in parentheses are percentages.

MDCT Findings in Patients with an Adverse Outcome Comparing the initial abnormal MDCT scans of those 6 patients with an adverse outcome to the remaining 6 patients with a favorable outcome, MDCT data revealed a markedly higher frequency of consolidation in patients with an adverse outcome (5/6, $83 \%$ vs. $2 / 6,33 \%)$. However, this difference was not statistical significant $(\mathrm{p}=$ $0.24)$.

Ground-glass opacities were detected in 5 of 6 MDCT datasets of patients with an adverse outcome as well as in 5 of 6 abnormal MDCT datasets of patients with a favorable outcome.

The combination of consolidations and ground-glass opacities with a bilateral affection of the lung parenchyma was found more frequently in the group of patients showing a severe clinical course (consolidation + groundglass opacities: $4 / 6,67 \%$ vs. $2 / 6,33 \%$; bilateral: $6 / 6,100 \%$ vs. $4 / 6,67 \%$ ) (fig. 2 ). 
Fig. 2. A 65-year-old woman with acute myeloid leukemia, who died 3 weeks after the onset of symptoms. Axial (a) and coronal MDCT scans (b) showing extensive airspace disease with bilateral multizonal consolidations (arrowheads) and groundglass opacities (arrow) affecting more than $50 \%$ of the lung parenchyma.


Tree-in-bud pattern was detected in half of the patients with a favorable outcome (3/6); it was not found in patients with an adverse course. All 6 patients with an adverse outcome showed bilateral and subpleural distribution of the observed abnormalities. Markedly more patients with an adverse outcome had an additional involvement of the central lung zones than did those patients who had a favorable clinical outcome $(5 / 6,83 \%$ vs. $1 / 6$, $17 \%)$. Involvement of more than $50 \%$ of the lung parenchyma was seen in $4(67 \%)$ patients with a poor outcome (fig. 2b), while only 1 patient with a mild course was found to have involvement of more than $50 \%$ of the lung parenchyma.

\section{Discussion}

Within the study population of 13 immunocompromised individuals with confirmed S-OIV infection, 12 (92\%) patients showed abnormal MDCT findings. Ground-glass opacities, frequently in a bilateral and subpleural distribution, were detected in the majority of the patients. Furthermore, consolidations were also commonly identified.

Half of the immunocompromised patients with initial abnormal findings revealed an adverse outcome. The bilateral distribution of consolidation with extensive involvement of the lung parenchyma correlated with an adverse outcome. These findings seem to be of some diagnostic value since all patients who revealed these abnormalities subsequently underwent life-supporting treatments such as mechanical ventilation and extracorporeal membrane oxygenation that required close monitoring.

Thoracic MDCT Findings of S-OIV in

Immunocompromised Patients
Additional infection of the respiratory tract is known as a major cause of morbidity and mortality in immunocompromised patients such as individuals with hematopoietic stem cell transplantation, solid organ transplant, HIV infection or long-term corticosteroid therapy $[7,11$, 12]. In a large US case series of hospitalized patients with S-OIV infection, underlying medical conditions were found in $67 \%$ of patients admitted to intensive care units, including immunosuppression (in 18\%), asthma or chronic obstructive pulmonary disease (in 28\%) [2].

Although the general radiological findings of thoracic S-OIV manifestation have been described in previous reports [13-18], hardly any attention was paid to the subset of immunocompromised patients $[8,9]$ who were described as a high-risk group for S-OIV infection and a more severe course $[7,19]$. Therefore, the present report has shown the importance of MDCT findings and their relevance in this special high-risk patient population.

Elicker et al. [9] described the MDCT findings in 8 immunocompromised patients with H1N1 infection, in which the most common findings were peripheral consolidation involving the lower lobes, ground-glass opacities, airway thickening/dilatation, centrilobular nodules and tree-in-bud pattern.

In our study, the predominant findings on MDCT were ground-glass opacities and consolidations, mostly bilateral with a peribronchovascular distribution. These predominant MDCT findings may be due to the ongoing pathological processes in the alveolar epithelium caused by S-OIV. Chan et al. [20] reported a high replication rate of S-OIV in bronchial epithelium, which can result in diffuse alveolar damage. However, the abnormal patterns identified in the present study in immunocompromised patients were concordant with those previously reported in immunocompetent individuals [14, 21]. 
However, an important difference between our findings and others is the rather high rate of patients with abnormal pulmonary findings at cross-sectional imaging and the high prevalence of a severe clinical course. These findings suggest that in immunocompromised patients an S-OIV infection can cause high morbidity and can affect the clinical course, resulting in severe pulmonary infection with acute respiratory distress syndrome and death. While $50 \%$ of our immunocompromised patients with initially abnormal radiologic findings had an adverse outcome, Aviram et al. [16] reported a rate of only $13 \%$ investigating immunocompetent patients exclusively. Hence, a severe outcome seems to be more frequent in the vulnerable group of patients suffering from immune deficiency.

The MDCT predictors of a severe clinical course were bilateral distribution of consolidation with extensive involvement of the lung parenchyma including the central lung zones. These findings seem to predict the progression of the S-OIV pulmonary infection to respiratory failure and ARDS requiring admission to the intensive care unit and the need for life-support treatments. Therefore, the early detection of these abnormalities at MDCT can help predict an adverse clinical outcome and to identify high-risk immunocompromised patients requiring close monitoring. In concordance with reports of immunocompromised patients having undergone imaging for various indications other than S-OIV [22], the MDCT finding of a tree-in-bud pattern was also demonstrated in this patient sample. This small-airway-related finding may provide radiological support for the findings of Munster et al. [23], who demonstrated the replication of S-OIV to occur not only in the trachea and bronchi but also in the bronchioles, using a ferret pathogenesis mod- el. Although bacteria are the most common cause of infectious bronchiolitis in immunocompromised patients, the differential diagnosis of this pattern does not only include mycobacterial or fungal infections but also viral disorders [24]. In our study focal areas of centrilobular nodular findings in a tree-in-bud pattern were frequently identified in patients with a mild course and may represent a mild localized inflammation.

This study has certain limitations. First, it was performed retrospectively, but a prospective study of immunocompromised patients with an acute onset is hardly feasible. Secondly, this report is limited by the heterogeneity of the study population consisting of different types of immunocompromised conditions. Thirdly, the assessment of an adverse outcome was based on only three parameters (the need for mechanical ventilation, extracorporeal membrane oxygenation, and death).

\section{Conclusion}

Initial MDCT findings were frequently abnormal in immunocompromised patients with suspected pulmonary involvement of confirmed S-OIV infection. Common initial findings were ground-glass opacities and consolidations predominantly with a bilateral distribution. Although the level of statistical significance was not reached, these results suggest that initial MDCT findings can help in predicting patient's outcome as bilateral consolidations, often combined with ground-glass opacities, with an extensive involvement of the lung parenchyma are frequently associated with an adverse clinical outcome.

\section{References}

1 World Health Organization: Global alert and response, pandemic (H1N1) 2009, frequently asked questions: what is phase 6 ? http://www.who.int/csr/disease/swineflu/ frequently_asked_questions/levels_pandemic_alert/en/index.html. Updated June 11, 2009, accessed November 12, 2009.

$\checkmark 2$ Jain S, Kamimoto L, Bramley AM, Schmitz AM, Benoit SR, Louie J, Sugerman DE, Druckenmiller JK, Ritger KA, Chugh R, et al: Hospitalized patients with $2009 \mathrm{H} 1 \mathrm{~N} 1 \mathrm{influ}-$ enza in the United States, April-June 2009. N Engl J Med 2009;361:1935-1944. $\checkmark 3$ Al-Baghli F, Al-Ateeqi W: Encephalitis-associated pandemic A (H1N1) 2009 in a Kuwaiti girl. Med Princ Pract 2011;20:191-195.

$\checkmark 4$ Louie JK, Acosta M, Winter K, Jean C, Gavali S, Schechter R, Vugia D, Harriman K, Matyas B, Glaser CA, et al: Factors associated with death or hospitalization due to pandemic 2009 influenza A (H1N1) infection in California. JAMA 2009;302:1896-1902.

$\checkmark 5$ Owayed AF, Husain EH, Al-Khabaz A, AlQattan HY, Al-Shammari N: Epidemiology and clinical presentation of pandemic influenza A (H1N1) among hospitalized children in Kuwait. Med Princ Pract 2012;21:254258 .
6 World Health Organization: Global Alert and Response (GAR). Pandemic (H1N1) 2009, update 112. http://www.who.int/csr/don/2010 08_06/en/index.html. August 6, 2010.

$\checkmark 7$ Lapinsky SE: H1N1 novel influenza A in pregnant and immunocompromised patients. Crit Care Med 2010;38:e52-e57.

$\checkmark 8$ Abbo L, Quartin A, Morris MI, Saigal G, Ariza-Heredia E, Mariani P, Rodriguez O, Muaoz-Price LS, Ferrada M, Ramee E, et al: Pulmonary imaging of pandemic influenza H1N1 infection: relationship between clinical presentation and disease burden on chest radiography and CT. Br J Radiol 2010;83: 645-651. 
9 Elicker BM, Schwartz BS, Liu C, Chen EC, Miller SA, Chiu CY, Webb WR: Thoracic CT findings of novel influenza A (H1N1) infection in immunocompromised patients. Emerg Radiol 2010;17:299-307.

10 Hansell DM, Bankier AA, MacMahon H, McLoud TC, Muller NL, Remy J: Fleischner Society: glossary of terms for thoracic imaging. Radiology 2008;246:697-722.

11 Kim YJ, Boeckh M, Englund JA: Community respiratory virus infections in immunocompromised patients: hematopoietic stem cell and solid organ transplant recipients, and individuals with human immunodeficiency virus infection. Semin Respir Crit Care Med 2007;28:222-242.

-12 Klein MB, Lu Y, DelBalso L, Cote S, Boivin $\mathrm{G}$ : Influenzavirus infection is a primary cause of febrile respiratory illness in HIVinfected adults, despite vaccination. Clin Infect Dis 2007;45:234-240.

13 Marchiori E, Zanetti G, D’Ippolito G, Verrastro, CG, Meirelles Gde S, Capobianco J, Rodrigues RS: Swine-origin influenza A (H1N1) viral infection: thoracic findings on CT. AJR Am J Roentgenol 2011;196:W723W728.

14 Agarwal PP, Cinti S, Kazerooni EA: Chest radiographic and $\mathrm{CT}$ findings in novel swineorigin influenza $\mathrm{A}(\mathrm{H} 1 \mathrm{~N} 1)$ virus (S-OIV) infection. AJR Am J Roentgenol 2009;193: 1488-1493.
15 Marchiori E, Zanetti G, Hochhegger B, Rodrigues RS, Fontes CA, Nobre LF, Mançano AD, Meirelles GS, Irion KL: High-resolution computed tomography findings from adult patients with Influenza A (H1N1) virus-associated pneumonia. Eur J Radiol 2010;74: 93-98.

16 Aviram G, Bar-Shai A, Sosna J, Rogowski O, Rosen G, Weinstein I, Steinvil A, Zimmerman O: H1N1 influenza: initial chest radiographic findings in helping predict patient outcome. Radiology 2010;255:252-259.

17 Lee EY, McAdam AJ, Chaudry G, Fishman MP, Zurakowski D, Boiselle PM: Swine-origin influenza a $(\mathrm{H} 1 \mathrm{~N} 1)$ viral infection in children: initial chest radiographic findings. Radiology 2010;254:934-941.

18 Shim SS, Kim Y, Ryu YJ: Novel influenza A (H1N1) infection: chest CT findings from 21 cases in Seoul, Korea. Clin Radiol 2011;66: 118-124.

19 Centers for Disease Control and Prevention (CDC): Updated Interim Recommendations: Special Considerations for Clinicians regarding $2009 \mathrm{H} 1 \mathrm{~N} 1$ Influenza in Severely Immunosuppressed Patients. http://www. cdc.gov/h1n1flu/immunosuppression/index.htm. December 16, 2009.
20 Chan MC, Chan RW, Yu WC, Ho CC, Yuen KM, Fong JH, Tang LL, Lai WW, Lo AC, Chui WH, et al: Tropism and innate host responses of the 2009 pandemic H1N1 influenza virus in ex vivo and in vitro cultures of human conjunctiva and respiratory tract. Am J Pathol 2010;176:1828-1840.

21 Henzler T, Meyer M, Kalenka A, Alb M, Schmid-Bindert G, Bartling S, Schoepf JU, Schoenberg SO, Fink C: Image findings of patients with H1N1 virus pneumonia and acute respiratory failure. Acad Radiol 2010; 17:681-685.

22 Franquet T, Lee KS, Muller NL: Thin-section CT findings in 32 immunocompromised patients with cytomegalovirus pneumonia who do not have AIDS. AJR Am J Roentgenol 2003;181:1059-1063.

23 Munster VJ, de Wit E, van den Brand JM, Herfst S, Schrauwen EJ, Bestebroer TM, van de Vijver D, Boucher CA, Koopmans M, Rimmelzwaan GF, et al: Pathogenesis and transmission of swine-origin 2009 A(H1N1) influenza virus in ferrets. Science 2009;325: 481-483.

24 Rossi SE, Franquet T, Volpacchio M, Gimenez A, Aguilar G: Tree-in-bud pattern at thin-section CT of the lungs: radiologicpathologic overview. Radiographics 2005; 25:789-801. 